\title{
ON THE LIOUVILLE THEOREM FOR HARMONIC MAPS
}

\author{
HYEONG IN CHOI
}

\begin{abstract}
Suppose $M$ and $N$ are complete Riemannian manifolds; $M$ with Ricci curvature bounded below by $-A, A \geqslant 0, N$ with sectional curvature bounded above by a positive constant $K$. Let $u: M \rightarrow N$ be a harmonic map such that $u(M) \subset$ $B_{R}\left(y_{0}\right)$. If $B_{R}\left(y_{0}\right)$ lies inside the cut locus of $y_{0}$ and $R<\pi / 2 \sqrt{K}$, then the energy density $e(u)$ of $u$ is bounded by a constant depending only on $A, K$ and $R$. If $A=0$, then $u$ is a constant map.
\end{abstract}

1. Introduction. Suppose $M$ is a complete Riemannian manifold with Ricci curvature bounded below by a constant $-A, A \geqslant 0$. Let $N$ be a complete Riemannian manifold with sectional curvature bounded above by a positive constant $K$. If $u: M \rightarrow N$ is a smooth map, we denote the energy density of $u$ by $e(u)$. We will prove the following theorem.

THEOREM. Let $u: M \rightarrow N$ be a harmonic map such that $u(M) \subset B_{R}\left(y_{0}\right)$ where $B_{R}\left(y_{0}\right)$ is the geodesic ball of radius $R$ centered at $y_{0}$ in $N$. If $B_{R}\left(y_{0}\right)$ lies inside the cut locus of $y_{0}$ and $R<\pi / 2 \sqrt{K}$, then $e(u)$ is bounded by a constant depending only on $A, K$ and $R$. If, furthermore, $A=0$, i.e., $M$ has nonnegative Ricci curvature, then $u$ is $a$ constant map.

Actually we have a more precise estimate for $e(u)$. (See formula (11) of $\S 3$.)

This type of theorem was first proved by S.-T. Yau [4] for harmonic functions. Generalizing Yau's theorem, S.-Y. Cheng [1] proved our theorem for harmonic maps assuming $N$ is simply connected with nonpositive sectional curvature. In fact, Cheng's result is more general in the sense that one could allow a certain growth of $B(a)$, where $B(a)=\sup \left\{d_{N}\left(u(x), y_{0}\right): x \in B_{a}\left(x_{0}\right)\right\}$ for some point $x_{0} \in M$, i.e., $\lim \sup _{a \rightarrow \infty} B(a) / a=0$.

On the other hand, the Dirichlet problem for harmonic maps was first solved by R. Hamilton in the case where $N$ is simply connected with nonpositive sectional curvature. This was generalized by Hildenbrandt, Kaul and Widman [3] who proved that the Dirichlet problem can be solved if we only assume $u(M) \subset B_{R}\left(y_{0}\right)$ with the same condition on $B_{R}\left(y_{0}\right)$ as in our theorem. In view of these two facts, it is natural to try to generalize Cheng's result to our situation. This is the motivation of our paper.

Our result is sharp as the following example shows. Let $S^{n}$ be the sphere of dimension $n$ with constant sectional curvature $K$. It is easy to see that the inclusion

Received by the editors May 18, 1981.

1980 Mathematics Subject Classification. Primary 53C99; Secondary 58E20, 35J60.

(c) 1982 American Mathematical Society 0002-9939/81/0000-11 18/\$02.00 
map $i: S^{n-1} \rightarrow S^{n}$ as the equator is harmonic, but clearly $S^{n-1}$ lies in the closed ball of radius $\pi / 2 \sqrt{K}$ centered at the north pole.

I would like to thank Professors $\mathrm{R}$. Schoen and $\mathrm{H}$. Wu for encouragement and many helpful discussions.

2. Preliminaries. We will employ Cheng's method of proof with minor modification. For the sake of completeness, we list some of the definitions and well-known formulas.

Choose local orthonormal frames $\left\{e_{\alpha}\right\}$ in a neighborhood of $p \in M$ and $\left\{f_{i}\right\}$ in a neighborhood of $u(p) \in N$. Let $\left\{\theta_{\alpha}\right\}$ and $\left\{w_{i}\right\}$ be the dual coframes of $\left\{e_{\alpha}\right\}$ and $\left\{f_{i}\right\}$ respectively. The connection forms $\left\{\theta_{\alpha \beta}\right\}$ and $\left\{w_{i j}\right\}$ are defined by the following equations:

$$
\begin{aligned}
d \theta_{\alpha}=\sum_{\beta} \theta_{\alpha \beta} \wedge \theta_{\beta}, & \theta_{\alpha \beta}+\theta_{\beta \alpha}=0, \\
d w_{i}=\sum_{j} w_{i j} \wedge w_{j}, & w_{i j}+w_{j i}=0 .
\end{aligned}
$$

Define $u_{i \alpha}$ by the equation

$$
u^{*} w_{i}=\sum_{\alpha} u_{i \alpha} \theta_{\alpha}
$$

The energy density $e(u)$ of $u$ defined to be $\Sigma_{i, \alpha} u_{i \alpha}^{2}$. Also define the covariant derivatives $u_{i \alpha \beta}$ by the equation

$$
\sum_{\beta} u_{i \alpha \beta} \theta_{\beta}=d u_{i \alpha}+\sum_{j} u_{j \alpha} u^{*} w_{j i}+\sum_{\beta} u_{i \beta} \theta_{\beta \alpha}
$$

It is well known that the condition, $\Sigma_{\alpha} u_{i \alpha \alpha}=0$ for all $i$, is equivalent to saying that $u$ is harmonic. The following Bochner type formula is also well known

$$
\frac{1}{2} \Delta e(u)=\sum_{i, \alpha, \beta} u_{i \alpha \beta}^{2}-\sum_{\substack{i, j, k, i, \alpha, \beta}} R_{i j k l} u_{i \alpha} u_{j \beta} u_{k \alpha} u_{l \beta}+\sum_{\alpha, \beta, i} K_{\alpha \beta} u_{i \alpha} u_{i \beta},
$$

where $R_{i j k l}$ is the curvature tensor of $N$, and $K_{\alpha \beta}$ is the Ricci tensor of $M$.

Let $\phi$ be a smooth function defined on $N$. Define $\phi_{i}$ and $\phi_{i j}$ by the following equations:

$$
d \phi=\sum_{i} \phi_{i} w_{i}, \quad \sum_{j} \phi_{i j} w_{j}=d \phi_{i}+\sum_{j} \phi_{j} w_{j i}
$$

One can also define the Hessian tensor $D^{2} \phi$ at a point $q \in N$ as follows. For any $X$, $Y \in N_{q}$, define $D^{2} \phi(X, Y)=X(Y \phi)-\left(D_{X} Y\right) \phi$, where $X$ and $Y$ are extended arbitrarily to vector fields in a neighborhood of $q . D_{X} Y$ denotes the covariant derivative of the Riemannian connection of $N$. It is easy to check $D^{2} \phi$ is a tensor and $D^{2} \phi\left(f_{i}, f_{j}\right)=\phi_{i j}$. We also have $\Delta_{N} \phi=\Sigma_{i} \phi_{i i}$.

Let $\phi$ be a strictly convex function defined in a neighborhood of a point $q=u(p) \in N, p \in M$. Suppose $\lambda(q)$ is the lower bound of the eigenvalues of the Hessian tensor $\left(\phi_{i j}\right)$ at $q$, then we have

$$
\left.\Delta_{M}(\phi \circ u)\right|_{p}=\sum_{i, j, \alpha} \phi_{i j} u_{i \alpha} u_{j \alpha} \geqslant \lambda(q) e(u) .
$$


It is also easy to check the following:

$$
\begin{gathered}
|d e(u)|^{2} \leqslant 4 e(u) \sum_{i, \alpha, \beta} u_{i \alpha \beta}^{2}, \\
|d(\phi \circ u)|^{2} \leqslant|d \phi|^{2} e(u) .
\end{gathered}
$$

3. Proof of the theorem. Multiplying the metric tensor by a suitable constant we may assume the upper bound of the sectional curvature of $N$ to be 1 throughout this section.

Let $\rho$ be the distance function from $y_{0}$ in $N$. Define $\phi=1-\cos \rho$.

Lemma. On $B_{R}\left(y_{0}\right),\left(\phi_{i j}\right) \geqslant(\cos \rho)\left(\delta_{i j}\right)$.

Proof. We will prove that the equality holds on $S^{n}$, the sphere of curvature $=1$; then the lemma follows by applying the Hessian comparison theorem of Greene and Wu [2, p. 19]. So for the rest of the proof of this lemma, we assume $N=S^{n}$. Applying Proposition 2.20 of [2, p. 30],

$$
D^{2} \rho=\frac{\cos \rho}{\sin \rho}\left(d S^{2}-d \rho \otimes d \rho\right)
$$

where $d S^{2}$ is the metric tensor of $N$. It is easy to check that $D^{2} \psi(\rho)=\psi^{\prime}(\rho) D^{2} \rho+$ $\psi^{\prime \prime}(\rho) d \rho \otimes d \rho$ for any smooth function $\psi: \mathbf{R} \rightarrow \mathbf{R}$. Thus for $\phi=1-\cos \rho$, we get $D^{2} \phi=(\cos \rho) d S^{2}$, i.e., $\left(\phi_{i j}\right)=(\cos \rho)\left(\delta_{i j}\right)$. Q.E.D.

Let $r$ be the distance function from $x_{0}$ in $M$. Since $R<\pi / 2$, we can choose a constant $b$ such that $\phi(R)<b<1$ on $B_{R}\left(y_{0}\right)$. Consider the function $\Phi=$ $\left(a^{2}-r^{2}\right)^{2} e(u) /(b-\phi \circ u)^{2}$ defined on the geodesic ball $B_{a}\left(x_{0}\right)$ in $M$. Since $\Phi$ vanishes on the boundary of $B_{a}\left(x_{0}\right)$, we may assume that $\Phi$ attains its maximum at an interior point $p$, and we may assume $e(u) \neq 0$ at $p$. We may also assume that $r$ is smooth near $p$ by the argument given in Cheng's paper [1]. Differentiating $\log \Phi$, we get, at $p$,

$$
\begin{gathered}
0=\frac{-2 d r^{2}}{a^{2}-r^{2}}+\frac{d e(u)}{e(u)}+\frac{2 d(\phi \circ u)}{b-\phi \circ u}, \\
0 \geqslant \frac{-2 \Delta r^{2}}{a^{2}-r^{2}}+\frac{-2\left|d r^{2}\right|^{2}}{\left(a^{2}-r^{2}\right)^{2}}+\frac{\Delta e(u)}{e(u)}-\frac{|d e(u)|^{2}}{e(u)^{2}} \\
+\frac{2 \Delta(\phi \circ u)}{b-\phi \circ u}+\frac{2|d(\phi \circ u)|^{2}}{(b-\phi \circ u)^{2}} .
\end{gathered}
$$

Using (1), (3) and the curvature bounds, we get

$$
\frac{\Delta e(u)}{e(u)} \geqslant \frac{1}{2} \frac{|d e(u)|^{2}}{e(u)^{2}}-2 e(u)-2 A .
$$

From (5), we get

$$
\frac{|d e(u)|^{2}}{e(u)^{2}} \leqslant \frac{4\left|d r^{2}\right|^{2}}{\left(a^{2}-r^{2}\right)^{2}}+\frac{8\left|d r^{2}\right||d(\phi \circ u)|}{\left(a^{2}-r^{2}\right)(b-\phi \circ u)}+\frac{4|d(\phi \circ u)|^{2}}{(b-\phi \circ u)^{2}} .
$$


From (2) and the lemma, we get $\Delta(\phi \circ u) \geqslant(\cos \rho) e(u)$. Applying this fact, (7) and (8) to (6), we obtain

$$
\begin{aligned}
0 \geqslant & \frac{-2 \Delta r^{2}}{a^{2}-r^{2}}-\frac{4\left|d r^{2}\right|^{2}}{\left(a^{2}-r^{2}\right)^{2}}-\frac{4\left|d r^{2}\right||d(\phi \circ u)|}{\left(a^{2}-r^{2}\right)(b-\phi \circ u)} \\
& -2 e(u)-2 A+\frac{2 \cos \rho}{b-\phi \circ u} e(u) .
\end{aligned}
$$

By the Laplacian comparison theorem [2, p. 26], there is a constant $C_{1}>0$ such that $\Delta r^{2} \leqslant C_{1}(1+r)$. By the Gauss lemma, $|d r|=1$. Also by (4) and the fact $|d \phi|=$ $\sin \rho \leqslant 1$, we get $|d(\phi \circ u)| \leqslant \sqrt{e(u)}$. It is easy to check that there is a constant $C_{2}>0$ such that

$$
-2+\frac{2 \cos \rho}{b-\phi \circ u}>C_{2}
$$

Hence we obtain from (9)

$$
\begin{aligned}
0 \geqslant & -2 A-\frac{2 C_{1}(1+r)}{a^{2}-r^{2}}-\frac{16 r^{2}}{\left(a^{2}-r^{2}\right)^{2}} \\
& -\frac{8 r}{\left(a^{2}-r^{2}\right)(b-\phi \circ u)} \sqrt{e(u)}+C_{2} e(u) .
\end{aligned}
$$

It is easy to see that if $a x^{2}-b x-c \leqslant 0$ with $a, b, c$ all positive, then

$$
x \leqslant \max \{2 b / a, 2 \sqrt{c / a}\} .
$$

Thus we get, at $p$,

$$
e(u) \leqslant 4 \max \left\{\frac{64 r^{2}}{C_{2}^{2}\left(a^{2}-r^{2}\right)^{2}(b-\phi \circ u)^{2}},\right.
$$

$$
\left.\frac{2 A}{C_{2}}+\frac{2 C_{1}(1+r)}{C_{2}\left(a^{2}-r^{2}\right)}+\frac{16 r^{2}}{C_{2}\left(a^{2}-r^{2}\right)^{2}}\right\} \text {. }
$$

From this we can obtain the upper bound of $\Phi$, and it can be easily concluded that (11) is true at every interior point of $B_{a}\left(x_{0}\right)$. Pick any point $x \in M$, thus $r(x)$ is fixed, and taking sufficiently large $a$ and letting $a \rightarrow \infty$, we have $e(u) \leqslant 8 A / C_{2}$. If $A=0$, then $e(u) \equiv 0$, i.e., $u$ is a constant map.

\section{REFERENCES}

I. S.-Y. Cheng, Liouville theorem for harmonic maps, Proc. Sympos. Pure Math., vol. 36, Amer. Math. Soc., Providence, R. I., 1980, pp. 147-151.

2. R. Greene and H. Wu, Function theory of manifolds which possess a pole, Lecture Notes in Math., vol. 699. Springer-Verlag, Berlin and New York, 1979.

3. S. Hildebrandt, H. Kaul and K.-O. Widman, An existence theorem for harmonic mappings of Riemannian manifolds, Acta Math. 138 (1977), 1-16.

4. S.-T. Yau, Harmonic functions on complete Riemannian manifolds, Comm. Pure Appl. Math. 28 (1975), 201-228.

Department of Mathematics, University of California, Berkeley, California 94720 